\title{
Análisis de la idoneidad didáctica de un proyecto de estadística diseñado e implementado en un contexto en riesgo de exclusión social
}

\author{
Ainhoa Berciano, Euskal Herriko Unibertsitatea \\ Ainhoa Subinas, Euskal Herriko Unibertsitatea \\ Jon Anasagasti, Euskal Herriko Unibertsitatea
}

\begin{abstract}
Análisis de la idoneidad didáctica de un proyecto de estadística diseñado e implementado en un contexto en riesgo de exclusión social

\section{Resumen}

La educación estadística debe responder al reto de cómo fomentar un pensamiento crítico en el alumnado. Este reto se singulariza en contextos en riesgo de exclusión social, por lo que convienen estudios que evalúen la idoneidad didáctica de distintos tipos de enseñanza. Nuestro interés se centra en analizar un proyecto de estadística específicamente diseñado para un contexto escolar de primaria socioeconómicamente complejo. Tras la implementación del proyecto en un aula de $5^{\circ}$ curso, hemos analizado: 1) el trabajo de contenidos curriculares y 2) la idoneidad didáctica de este trabajo de acuerdo a dimensiones teóricas preestablecidas. Concluimos que se debe atender al equilibrio entre las dimensiones menos representadas, denominadas epistémica y mediacional, con respecto a las dimensiones denominadas ecológica, cognitiva, afectiva e interaccional.
\end{abstract}

Palabras clave. Contenidos estadísticos; Aprendizaje Basado en Proyectos; Enfoque Ontosemiótico; contexto en riesgo de exclusión social; Educación Primaria.

Analysis of the didactic suitability of a statistical project designed and implemented in a context at risk of social exclusion

\section{Abstract}

Statistical education must respond to the challenge of how to foster critical thinking in students. Such a challenge becomes singularized in contexts at risk of social exclusion, and hence studies that evaluate the didactic suitability of different types of education are key. Our interest is focused on analyzing a statistical project specifically designed for a socioeconomically complex school context of primary education. After the project implementation in a 5th grade classroom, we analyzed: 1) the work around curricular contents and 2) its didactic suitability according to theoretically-established dimensions. We conclude about the need to address a certain balance amongst the least represented dimensions, named herein epistemic and mediational, in line with those named ecological, cognitive and interactional.

Keywords. Statistical contents; Project Based Learning; Ontosemiotic Approach; context at risk of social exclusion; Primary Education.

\section{Introducción}

Una preocupación que han trasladado los resultados de las pruebas TIMSS y PISA es la existencia de una fuerte correlación de los resultados del alumnado con su entorno y situación familiar y, en particular, con la variable socioeconómica (INEE, 2015; OECD, 2019). PISA muestra una relación positiva entre situaciones socioeconómicas menos favorecidas y puntuaciones más negativas en los resultados de las pruebas. Esto también se ve reflejado en el estudio de las competencias matemática y estadística (ISEIIVEI, 2014). Esta relación implica que, cuando el contexto socioeconómico negativo es 
generalizado y afecta a gran parte del alumnado de la clase, la realidad educativa se vuelve más compleja. Ante esto, la educación particularmente matemática debe plantear propuestas de intervención que ayuden al aprendizaje de todos los grupos de alumnado. Es necesario, por ejemplo, diseñar proyectos que generen dinámicas de participación y de comprensión de los significados en situaciones de interacción y de discusión en las aulas, a modo de facilitar la producción de significado matemático con sentido. En el área de investigación en educación matemática, tal como destaca Planas (2010), hay interés por aportar resultados y conocimiento acerca de entornos de aprendizaje inclusivos que sean a la vez matemáticamente potentes para todos los grupos.

Dicho lo anterior, en este artículo explicamos una investigación orientada a evaluar la pertinencia y validez de un proyecto específicamente diseñado e implementado para trabajar contenidos de estadística en $5^{\circ}$ curso de la etapa de educación primaria en un centro con alumnado en riesgo de exclusión social. Analizamos el proyecto según: 1) el trabajo de contenidos del currículum y 2) su idoneidad de acuerdo a las dimensiones definidas desde el denominado Enfoque Ontosemiótico (EOS).

\section{Antecedentes y marco conceptual}

Entendemos por entorno de exclusión social aquel marcado por la vulnerabilidad social. Esta definición trata de representar al conjunto de personas o comunidades que participan en la sociedad desde la periferia. En un entorno de estas características la educación matemática adquiere un papel fundamental; es materia instrumental y constante a lo largo de la escolarización, por lo que su potencialidad de influir en los itinerarios académicos es superior a otras materias, aunque esta es una razón técnica todavía no asociada a las condiciones y oportunidades del aprendizaje.

Dentro de las metodologías de enseñanza y aprendizaje en el aula de matemáticas, el Aprendizaje Basado en Proyectos (ABP) ofrece condiciones y oportunidades de aprendizaje que se adaptan bien a las necesidades del alumnado también en entornos desfavorecidos por estar centrado en tareas, relacionarse con otras disciplinas y mostrar versatilidad en cuanto a ritmos de trabajo (Pozuelos y Rodriguez, 2008). Esta modalidad de enseñanza y aprendizaje centrada en tareas promueve el aprendizaje individual y autónomo dentro de un plan de trabajo definido por objetivos y procedimientos (GarcíaValcárcel y Basilotta, 2017). Otro aspecto del ABP es la relación con el aprendizaje significativo (Thomas, 2000); los proyectos proponen situaciones relacionadas con la vida real, de manera que el aprendizaje procedimental y los contenidos se contextualizan y significan. En palabras de Mergendoller, Maxwell y Bellisimo (2006), la evidencia empírica sugiere que: "el ABP tiene un efecto positivo en la adquisición de conocimientos por parte de los estudiantes, el desarrollo de habilidades como la colaboración, el pensamiento crítico y la resolución de problemas" (p. 115). Además, la participación en actividades de ABP refuerza el compromiso con el aprendizaje.

Batanero y Díaz (2011) afirman que hay una estrecha relación teórica entre aprendizaje estadístico y aprendizaje basado en proyectos. Estas autoras destacan la necesidad de plantear la educación estadística desde el ABP, argumentando que los objetivos de la enseñanza de la estadística (diseñar investigaciones, recoger datos de investigación, representar datos en tablas, comparar representaciones y obtener conclusiones de la investigación realizada) coinciden en gran medida con los principios metodológicos del ABP (aprendizaje situado, resolución de un proyecto real, planteamiento de cuestiones e hipótesis iniciales, discusión de hipótesis y resultados utilizando métodos científicos). Esta relación teórica ha dado lugar a dos enfoques de investigación: 1) uno centrado en 
analizar y medir la repercusión del ABP en contextos reales de aula de estadística y 2) otro focalizado en determinar el cumplimiento real del diseño ABP acorde a marcos teóricos de enseñanza y aprendizaje de la matemática.

Con respecto a la primera línea de investigación, destacamos trabajos en todas las etapas educativas. En niveles universitarios, Tarmizi y Bayat (2010) y Anasagasti y Berciano (2017) muestran que la implementación del ABP mejora la motivación del alumnado, mientras que Subinas y Berciano (2019) apuntan a una evolución positiva de la afectividad. Para etapas educativas anteriores, el currículo educativo alude a la necesidad de trabajar la estadística de modo contextualizado con significación de contenidos en "conexión con actividades que implican otras áreas de conocimiento" (Decreto 236/2015, 2016, p.170), destacando el valor de los contenidos estadísticos como herramienta docente para potenciar el trabajo en equipo. Batanero, Díaz, Contreras y Roa (2013) ponen de relieve el papel del trabajo con proyectos en el desarrollo del sentido estadístico del estudiante de forma progresiva desde primaria. En esta línea, investigaciones sobre cursos iniciales de educación secundaria afirman que la educación estadística basada en ABP muestra ventajas frente a metodologías tradicionales a nivel competencial (Koparan y Güven, 2014) y actitudinal (Siswono, Hartono y Kohar, 2018).

Con respecto a la segunda línea de investigación, para el diseño ABP acorde a marcos teóricos de enseñanza y aprendizaje de la matemática, nuestro marco de referencia es el Enfoque Ontosemiótico (EOS). La elección del EOS (Godino, Batanero y Font, 2007) se debe a la gran variedad de investigaciones llevadas a cabo en el ámbito de la enseñanza de la estadística, entre ellas, la variedad que usan la herramienta de la idoneidad didáctica para mostrar su aplicabilidad tanto en evaluar implementaciones de aula como en formaciones de profesorado de matemáticas (Breda, Pino-Fan y Font, 2017; Font, Planas y Godino, 2010; Nogueira, Fernández y Rodríguez Vivero, 2015; Ramos, Fagúndez y Castells, 2009).

El Enfoque Ontosemiótico (EOS) establece la idoneidad didáctica como el criterio sistémico de pertinencia o adecuación de un proceso de instrucción al proyecto educativo, cuyo principal indicador empírico es la adaptación entre significados personales logrados y significados institucionales pretendidos o implementados (Godino, Wilhelmi y Bencomo, 2005; Godino, Contreras y Font, 2006). El logro de una idoneidad didáctica alta de un proceso de instrucción requiere tener en cuenta de manera sistémica seis dimensiones o facetas (Godino, Batanero y Font, 2007):

1) Idoneidad epistémica: correspondencia entre los contenidos o significados implementados y los de referencia.

2) Idoneidad ecológica: adecuación del proceso de enseñanza y aprendizaje al entorno social y proyecto de centro.

3) Idoneidad cognitiva: adecuación de los significados implementados a los conocimientos previos y diversidad de características del alumnado.

4) Idoneidad afectiva: implicación del alumnado en el proceso implementado.

5) Idoneidad interaccional: potencialidad del proceso enseñanza-aprendizaje para identificar y resolver posibles conflictos semióticos.

6) Idoneidad mediacional: disponibilidad y adecuación de medios para el desarrollo del proceso de instrucción.

Cada una de estas facetas se conforma por diferentes componentes (ver Tabla 1); para cada componente se propone un conjunto de indicadores de idoneidad con el fin de garantizar su consecución (Godino, 2013). 
Tabla 1. Componentes asociadas a cada dimensión de la idoneidad didáctica

\begin{tabular}{|c|c|}
\hline DIMENSIONES & COMPONENTES \\
\hline Epistémica & Situaciones problema, lenguajes, reglas, argumentos, relaciones \\
\hline Ecológica & $\begin{array}{l}\text { Innovación, adaptación sociocultural, curricular y profesional, } \\
\text { conexiones intra e interdisciplinares }\end{array}$ \\
\hline Cognitiva & $\begin{array}{l}\text { Conocimientos previos, adaptación curricular a diferencias } \\
\text { individuales, aprendizajes }\end{array}$ \\
\hline Afectiva & Intereses, actitudes, emociones \\
\hline Interaccional & Interacción docente-discente, interacción entre discentes, autonomía \\
\hline Mediacional & $\begin{array}{l}\text { Recursos materiales, número de estudiantes, condiciones de aula, } \\
\text { tiempo para la enseñanza y el aprendizaje }\end{array}$ \\
\hline
\end{tabular}

La caracterización del grado de idoneidad didáctica de una práctica docente matemática supone un análisis multidimensional complejo. Vemos ejemplos en investigaciones acerca de la enseñanza y aprendizaje de la estadística y probabilidad que indican la necesidad de mejorar la formación en la componente mediacional del futuro profesorado de estadística (Arteaga, Batanero y Gea, 2017), o la importancia del conocimiento de los indicadores asociados a la idoneidad afectiva en la enseñanza de la probabilidad (Beltrán-Pellicer y Godino, 2017). Sin embargo, son escasos los trabajos que analizan las repercusiones de la educación estadística usando la metodología ABP en Educación Primaria, sin estudios enmarcados en contextos de riesgo de exclusión desde el EOS.

\section{Objetivos y métodos}

Nuestro objetivo fundamental es evaluar y analizar la idoneidad didáctica de un proyecto de estadística titulado "Conocer el Mercado", diseñado e implementado para un aula de $5^{\circ}$ de Primaria con alto riesgo de exclusión social. Los dos objetivos específicos son: 1) Evaluar la idoneidad del proyecto atendiendo a qué actividades desarrollan los contenidos a tratar en el currículo de Educación Primaria y en qué grado. 2) Evaluar la idoneidad didáctica del proyecto mediante el análisis de la prevalencia de las dimensiones establecidas según el EOS para el conjunto de las actividades.

Nuestra investigación adopta un enfoque interpretativo de diseño (Molina, Castro, Molina y Castro, 2011). Investigadoras y docente colaboramos previamente al desarrollo de este proyecto en actividades (Subinas y Berciano, 2019) para: a) Describir rasgos del grupo e identificar prioridades en la gestión de aula y necesidades específicas del alumnado. b) Identificar conocimientos previos de manera global según conocimiento en matemáticas, vocabulario y riqueza en argumentación e interés en el aprendizaje. c) Vincular investigadora y alumnado a fin de facilitar la intervención e investigación. Desde este enfoque, se ha llevado a cabo un análisis cualitativo de las actividades desarrolladas, para luego realizar un análisis cuantitativo que muestre el peso relativo de cada dimensión de un proceso de instrucción y así comprobar la idoneidad didáctica.

Para el análisis de las actividades diseñadas e implementadas se han tenido en cuenta distintas herramientas, que pasamos a describir. En cuanto al primer objetivo, se consideran indicadores los contenidos específicos del currículo acerca del aprendizaje estadístico del primer y segundo ciclos de E.P., esto es, de los cursos $1^{\circ}, 2^{\circ}$ y $3^{\circ}, y^{\circ}, 5^{\circ}$ y $6^{\circ}$ de E.P. respectivamente (Decreto 236/2015, 2016). Se amplían a las dos etapas debido a la diferencia en conocimientos previos y necesidades específicas de aprendizaje de cada discente $\mathrm{y}$, por tanto se debe hacer una adaptación de contenido a cada estudiante. A continuación, se enumeran de acuerdo al orden de aparición en el decreto los siguientes elementos (Decreto 236/2015, 2016, anexo 2, pp.159-165): 
- Primer ciclo (cursos $1^{\circ}, 2^{\circ}$ y $3^{\circ}$ ): recogida de datos sobre objetos, fenómenos y situaciones con técnicas de muestreo (C11); elaboración de cuadros de doble entrada de datos sobre objetos, fenómenos y situaciones familiares (C12); elaboración de gráficos de barras con datos relativos a objetos, fenómenos y situaciones del entorno (C13); lectura e interpretación de textos numéricos en forma de tablas de doble entrada de uso habitual en la vida cotidiana (C14); lectura e interpretación de gráficos de barras relativas a fenómenos familiares (C15).

- Segundo ciclo (cursos $4^{\circ}, 5^{\circ}$ y $6^{\circ}$ ): recogida y clasificación de datos cualitativos y cuantitativos relativos a objetos, fenómenos y situaciones del entorno, utilizando técnicas elementales de encuesta, observación y medición (C21); interpretación y construcción de tablas de frecuencias absolutas y relativas (C22); realización e interpretación de gráficos sencillos para representar la información: diagramas de barras, pictogramas poligonales y sectoriales (C23); iniciación intuitiva a las medidas de centralización: media aritmética, moda y rango (C24); análisis crítico de las informaciones que se presentan mediante gráficos estadísticos (C25).

Para evaluar el segundo objetivo se han tenido en cuenta las seis dimensiones que caracterizan un proceso de instrucción según el EOS, sus componentes descritas en la Tabla 1 y los indicadores asociados a cada una de las componentes. La implementación del proyecto se ha llevado a cabo en un centro de Bilbao, en uno de los barrios más deprimidos de la ciudad, y se ha trabajado en un aula de $5^{\circ}$ de Educación Primaria con 15 estudiantes (8 niñas y 7 niños). La escuela presenta alta diversidad de alumnado y alto porcentaje de familias en situación de riesgo de exclusión social, etnias minoritarias, inmigración escolarizada a mitad de curso, dificultades con el idioma (castellano y sobre todo euskera) y porcentaje alto de riesgo de abandono temprano de la educación.

\section{Diseño y desarrollo del proyecto y de las actividades}

El proyecto que se evalúa implementa aspectos de diferentes disciplinas y provocar conflicto cognitivo para suscitar interés en el contenido. Se abordan conceptos de demografía en el área de ciencias sociales y elementos básicos para el desarrollo del "sentido estadístico" en matemáticas (Berciano, Subinas y Anasagasti, 2020). Esta elección se debe a que el currículo para ciencias sociales establece que "La idea de globalidad debe guiarnos en esta etapa [de primaria] y por consiguiente en el área que nos incumbe, sabiendo integrar los diferentes contenidos en torno a la experimentación, investigación, trabajos de campo, salidas, [...]" (Decreto 236/2015, 2016:179). Siguiendo las indicaciones de Gómez y Martínez (1997), el objetivo fundamental del proyecto es promover conocimientos científicos de ciencias sociales, aprendizajes de resolución de cuestiones prácticas y de desarrollo de pensamiento crítico, reflexivo y transformador. Para esto, las herramientas estadísticas resultarán imprescindibles.

El proyecto de estadística diseñado se plantea como respuesta al problema generado en la unidad didáctica de la asignatura de ciencias sociales de quinto curso de educación primaria: la población. Esta unidad didáctica se centra en trabajar conceptos asociados a la descripción de la población: división población activa-no activa, sectores laborales, etc. Atendiendo al esquema de Wild y Pfannkuch (1999), se ha realizado una adaptación de las fases de aprendizaje del ABP al contexto de aprendizaje. Este hecho ha llevado a plantear tres bloques, con cinco fases y doce acciones concretas (Tabla 2).

Tabla 2. Bloques, fases y acciones definidas en el proyecto

\begin{tabular}{lll}
\hline Bloques & Fases & Acciones \\
\hline
\end{tabular}




\begin{tabular}{|c|c|c|c|}
\hline \multirow[t]{4}{*}{ Problema } & \multirow[t]{2}{*}{ Plantear problema } & $\begin{array}{l}\text { A1 } \\
\text { A2 }\end{array}$ & $\begin{array}{l}\text { Presentación de la unidad "La población" } \\
\text { Discusión en clase: ¿Cómo es la población en } \\
\text { nuestro entorno? }\end{array}$ \\
\hline & & A3 & $\begin{array}{l}\text { Acotación del proyecto: "Conozcamos el } \\
\text { mercado" }\end{array}$ \\
\hline & \multirow[t]{2}{*}{ Plantear preguntas } & A4 & Diseño de las preguntas \\
\hline & & A5 & Diseño de la encuesta para la visita \\
\hline \multirow[t]{6}{*}{ Datos } & Recoger datos & A6 & Visita al mercado y realización de encuestas \\
\hline & \multirow{5}{*}{$\begin{array}{l}\text { Organizar e } \\
\text { interpretar datos }\end{array}$} & A7 & Lectura de gráficos \\
\hline & & A8 & Recuento de datos \\
\hline & & A9 & Aproximación a la construcción de gráficos \\
\hline & & A 10 & Corrección y rediseño de gráficos \\
\hline & & A11 & Interpretación de datos \\
\hline Conclusiones & Generar informe & A12 & Construcción del mural \\
\hline
\end{tabular}

En la siguiente tabla detallamos brevemente la implementación con la descripción de las tareas involucradas por bloques, sus objetivos y metodología usada (Tabla 3):

Tabla 3. Implementación de las acciones, objetivos, metodología y número de sesiones

\begin{tabular}{llll}
\hline Acción & Objetivo & $\begin{array}{l}\text { Metodología- } \\
\text { agrupación }\end{array}$ & $\begin{array}{l}\mathrm{N}^{\mathbf{o}} \\
\text { Sesiones }\end{array}$ \\
\hline A1-A2 & Identificar los conocimientos previos del alumnado & $\begin{array}{l}\text { Interrogativa } \\
\text { Asamblea }\end{array}$ & 2 \\
A3 & $\begin{array}{l}\text { Acotar el entorno de investigación por medio de } \\
\text { preguntas de interés }\end{array}$ & Asamblea & 1 \\
\hline A4-A5 & $\begin{array}{l}\text { Consensuar las preguntas del proyecto asociadas a } \\
\text { variables cualitativas y cuantitativas y trabajar la } \\
\text { competencia escrita, el euskera }\end{array}$ & $\begin{array}{l}\text { Parejas y grupo- } \\
\text { clase }\end{array}$ & 2 \\
\hline A6 & Realizar la recogida de datos & Parejas & 1.5 \\
\hline A7 & $\begin{array}{l}\text { Ver la necesidad de organizar las respuestas } \\
\text { recogidas de modo visual }\end{array}$ & $\begin{array}{l}\text { Individual- } \\
\text { grupal }\end{array}$ & 1 \\
A8 & $\begin{array}{l}\text { Fomentar la implicación, y que la distribución } \\
\text { promueva la idea de interdependencia }\end{array}$ & $\begin{array}{l}\text { Aula en forma } \\
\text { de U }\end{array}$ & 1 \\
A9 & $\begin{array}{l}\text { Fomentar la interdependencia en el proceso y así la } \\
\text { implicación del alumnado }\end{array}$ & $\begin{array}{l}\text { Aula en forma } \\
\text { de U }\end{array}$ & 1 \\
A10 & $\begin{array}{l}\text { Analizar y corregir los gráficos realizados } \\
\text { Poner en común la interpretación de los gráficos } \\
\text { A11 }\end{array}$ & $\begin{array}{l}\text { Parejas } \\
\text { Grupal }\end{array}$ & 1 \\
\hline A12 & Resituados y compararlo con las hipótesis originales & 1 \\
\hline
\end{tabular}

En las actividades A1 y A2 los contenidos tratados están asociados a la demografía, mediante variables principalmente cualitativas: población activa-no activa; personas sin empleo, retiradas, menores, mayores; sectores de población; ¿Cómo evoluciona nuestra ciudad?; ¿Dónde podemos conocer personas del primer sector? Las ciudades y el campo: entorno rural y urbano; vocabulario asociado. Igualmente, en A3, se plantean las preguntas de interés del proyecto "Las poblaciones son muy diversas... janalicemos nuestro entorno más cercano: el mercado!", priorizando las variables de análisis (personas que van, empleos, cuánto gastan, etc.), con su posterior clasificación según su tipología en las actividades A4 y A5 (Figura 1). 


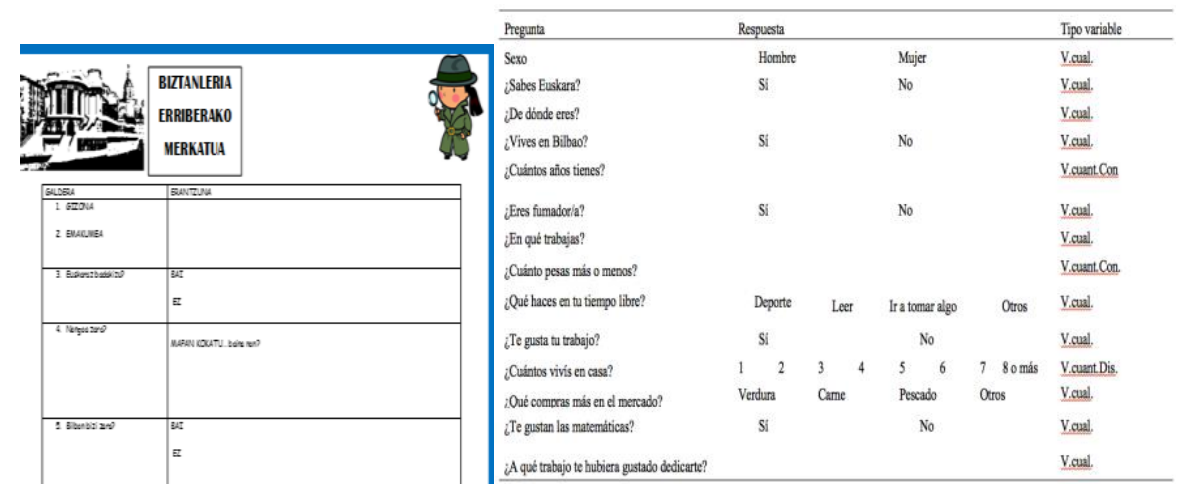

Figura 1. Diseño de encuesta. Cuestiones que incluyen los tipos de variables a trabajar: cualitativa, cuantitativa discreta y continua (traducción a la derecha)

En A6, cada pareja realiza la recogida de datos, con una muestra no inferior a 10 personas. A la vuelta, en el centro, se trabaja cómo describir globalmente los datos. Se construye una primera aproximación cualitativa e intercambio de impresiones y así poder aunar las respuestas de todos los participantes.

Para organizar los datos recogidos, A7 consta de dos partes: primera, para trabajar la lectura de gráficos en el aula, se introducen los diagramas de barras y su potencialidad como herramienta de representación de datos; segunda, para obtener una idea más exacta del nivel de interpretación del alumnado se exponen dos diagramas similares, pero con diferentes escalas, que aparentemente presentan situaciones análogas sobre dos ciudades (Figura 2). Se crea una batería de preguntas siguiendo los niveles de Friel, Curcio y Bright (2001), para la lectura y escritura de gráficos que incluyen desde "extracción de datos", hasta "lectura detrás de los datos" (Arteaga, Batanero y Contreras, 2011).
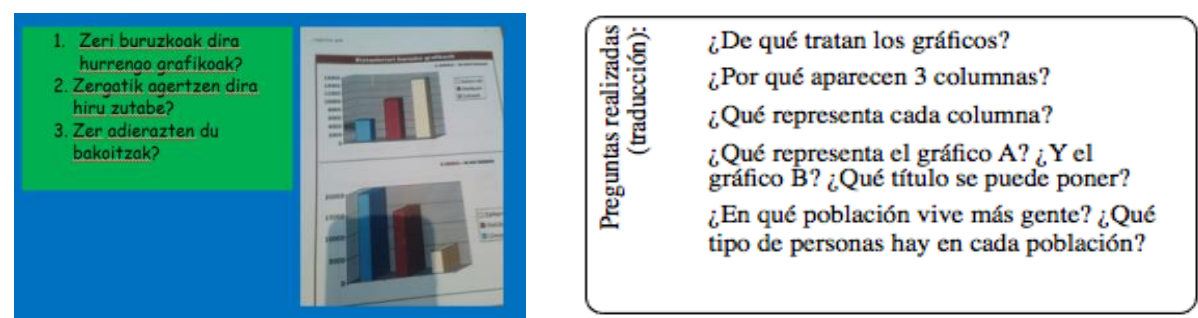

Figura 2. Cuestionario sobre lectura de gráficos (traducción a su derecha). Comparación de gráficos e interpretación de datos

Para el recuento de datos (A8), se realizan dos ajustes: 1) se crean parejas de estudiantes con conocimientos estadísticos parecidos, 2) se distribuyen las variables atendiendo a competencias del alumnado para que únicamente analicen las variables que aparezcan en su plantilla (Figura 3). Así, las variables cualitativas se reparten entre las parejas con más dificultades y las cuantitativas continuas entre las otras.

En la construcción de los gráficos (A9), cada estudiante representa una variable. Se provee de material menos usual: folios, cartulinas y pegatinas de colores. Se realiza una primera aproximación: pegatina en forma cuadrada por cada 5 elementos, construyendo una tabla, similar a un diagrama de barras más intuitiva. Tras la construcción de los gráficos, se corrigen y se pide al alumnado que realice otro (A10), adaptado a su competencia estadística: quien tiene mayores dificultades realiza el gráfico de forma similar, pero aquellos que han entendido bien la construcción de gráficos, dibujan un diagrama de barras o histograma, escriben la escala en el eje de ordenadas y los datos en el eje horizontal y realizan una pequeña descripción de los resultados, que luego exponen en común comparando las impresiones iniciales con los resultados obtenidos (A11). 


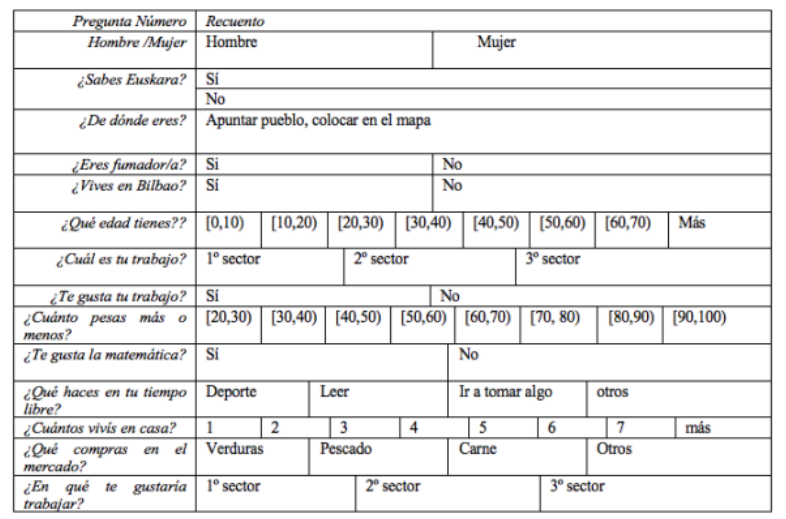

Figura 3. Plantilla usada para el recuento de datos (traducción)

La última sesión A12 (de hora y media) se dedica a la creación de un mural, proyectando la imagen del mercado con la pantalla digital, se calca y se decora con los gráficos realizados por cada estudiante (Figura 4).

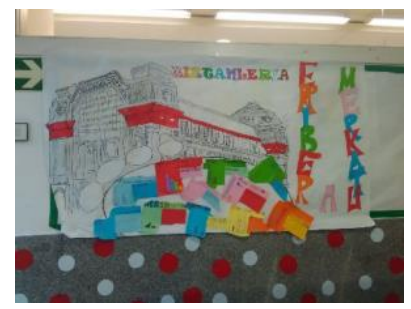

Figura 4. Creación del mural final e interpretación global del proyecto

\section{Análisis y discusión de datos}

A continuación, mostramos los resultados acordes a los dos objetivos de la investigación, empezando por el análisis de las acciones de acuerdo a los contenidos comprendidos en el currículum. Aquí, se observa el desarrollo de los contenidos en el currículum en las diferentes actividades (Tablas 4 y 5 ).

Tabla 4. Indicadores para actividades con contenidos de primer ciclo

\begin{tabular}{rrrrrr}
\hline & C11 & C12 & C13 & C14 & C15 \\
\hline A1 & & & & \\
A2 & $\mathrm{x}$ & & & \\
A3 & $\mathrm{x}$ & & & \\
A4 & $\mathrm{x}$ & & & \\
A5 & $\mathrm{x}$ & & & \\
A6 & $\mathrm{x}$ & & & & \\
A7 & & & & & \\
A8 & $\mathrm{x}$ & & & \\
A9 & & $\mathrm{x}$ & & \\
A10 & & $\mathrm{x}$ & & \\
A11 & & & $\mathrm{X}$ \\
\hline A12 & & & \\
\hline
\end{tabular}

Tabla 5. Indicadores para actividades con contenidos de segundo ciclo

\begin{tabular}{llllll}
\hline & C21 & C22 & C23 & C24 & C25 \\
\hline A1 & $X$ & & & & \\
A2 & $X$ & & & \\
A3 & $X$ & & & \\
A4 & X & & & \\
A5 & X & & & & \\
\hline
\end{tabular}




\begin{tabular}{rll}
\hline A6 & $x$ & \\
A7 & $x$ & \\
A8 & $x$ & $x$ \\
A9 & & $x$ \\
A10 & & $x$ \\
A11 & & $x$ \\
A12 & & \\
\hline
\end{tabular}

Este análisis indica que el contenido C11 se trabaja en las cinco primeras actividades, mientras que los contenidos $\mathrm{C} 12, \mathrm{C} 13, \mathrm{C} 14$ y C15 sólo se trabajan en una o dos actividades (Tabla 4). Un análisis relativo a los contenidos del segundo ciclo muestra que $\mathrm{C} 21$ se trabaja en las cinco primeras actividades, C22 sólo en dos actividades, C23 en cuatro y C24 y C25 no se trabajan (Tabla 5).

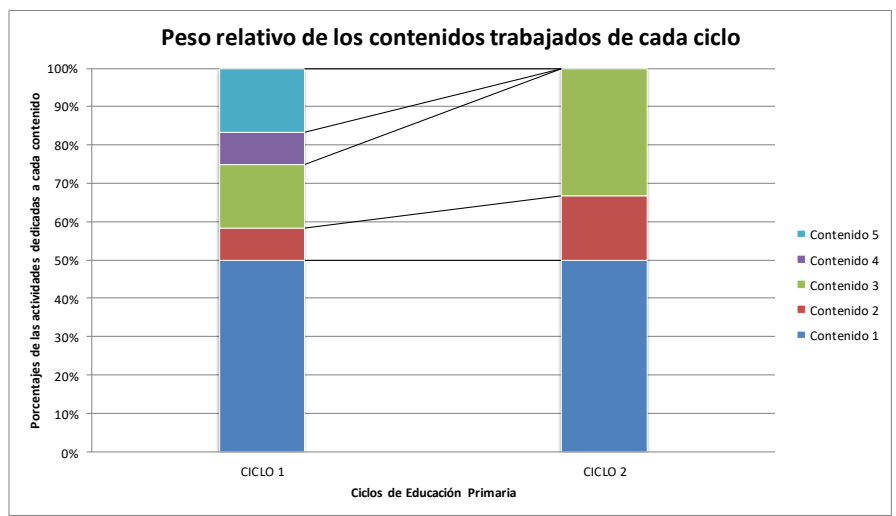

Gráfico 1. Peso relativo de los contenidos trabajados de cada ciclo

Si denotamos por Contenido 1 al contenido C11 del primer ciclo y al contenido C21 del segundo (e igualmente Contenido 2 para C12 y C22, etc.) (Gráfico 1), destacamos la prevalencia en la recogida de datos y procesos de muestreo y realización de gráficos, de manera que quedan contenidos, como la interpretación crítica de datos o cálculos estadísticos, sin trabajar. A fin de evaluar las acciones de acuerdo a las dimensiones de la idoneidad didáctica, hemos realizado un análisis del diseño y proceso de instrucción, observando la evidencia/ausencia de dichas componentes en el conjunto de actividades.

\section{Dimensión epistémica}

- Situación-problema: se problematiza la unidad didáctica sobre la población, la dificultad para describir sus características y la necesidad de una "metodología de investigación" para obtener datos.

- Lenguaje y reglas: se proponen gráficos de distinta complejidad para interpretación.

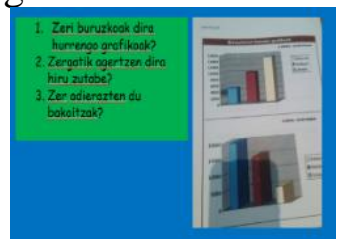

Figura 5. Lectura de gráficos de diferente complejidad

- Argumentos y relaciones: si bien las explicaciones se adaptan al nivel del alumnado, las explicaciones, definiciones y proposiciones, tanto como las conexiones tratan de reducirse lo máximo posible y acercar el trabajo de manera más práctica tratando de evitar el conocimiento más abstracto.

La dimensión epistémica se centra en el desarrollo de la problematización del proyecto. Un grado alto de idoneidad supondría promover espacios para la argumentación y la 
conjetura, y a su vez desarrollar diversos modos de expresión en que incluir definiciones y argumentaciones de tipo más abstracto. Este segundo aspecto no se ha llevado a cabo por dificultades con la expresión tanto en el idioma, como en riqueza de expresión y por la limitada capacidad de escucha en el desarrollo de este tipo de tareas.

\section{Dimensión cognitiva}

- Conocimientos previos: se parte sobre las ideas estereotipo previas del alumnado sobre los elementos vinculados al área de CC.SS.

- Adaptaciones curriculares a diferencias individuales: la adaptación del material es global, pero a su vez se atienden diferencias al asignar tareas (variables cualitativas o cuantitativa continuas a alumnos según más o menos dificultades).

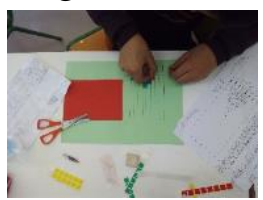

Figura 6. Realización de gráficos con pegatinas para representar frecuencias absolutas

- Aprendizajes: la evaluación es global y continua. Se observa la implicación del alumnado en las tareas y el no abandono de la misma se considera fundamental.

\section{Dimensión ecológica}

- Adaptación al currículo: el diseño del proyecto sigue las directrices curriculares, aunque se observa que dada la diversidad del alumnado es difícil desarrollar aquellos especificados para su ciclo en su totalidad.

- Adaptación socioprofesional y cultural: el contexto de desarrollo del proyecto forma parte del entorno más cercano del alumnado: su propio barrio, su mercado y su gente.

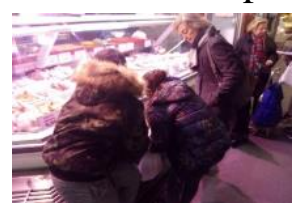

Figura 7. Visita al mercado del barrio y realización de la encuesta

- Conexiones intra e interdisciplinares: el proyecto tiene un cariz interdisciplinar con el trabajo de la expresión escrita (redacción de preguntas), la competencia emprendedora, cívica, conceptos de ciencias sociales y elementos de expresión artística (construcción mural).

- Apertura a la innovación didáctica: la propia realización del aprendizaje basado en proyectos aplicado al estudio de la estadística.

\section{Dimensión afectiva}

- Intereses, necesidades y actitudes: los cuestionarios iniciales se construyen a partir de intereses del alumnado. La tarea general se compone de sub-tareas diferenciadas donde participan conjuntamente. Cada tarea (gráfico de cada variable) está destinado por afinidad con cada alumno.

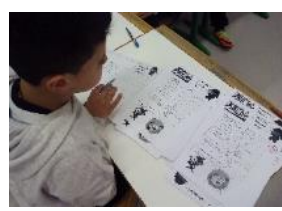

Figura 8. Resolución de ejercicio por parte de estudiante 
- Emociones: el conjunto de la clase está implicado en la tarea, todo el grupo finalizó su gráfico y lo incluyó en el mural.

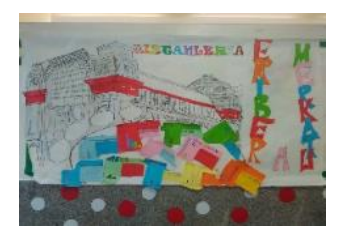

Figura 9. Muestra del mural final con las aportaciones individuales

La evidencia fundamental del éxito del trabajo realizado en las dimensiones epistémica, cognitiva y ecológica puede observarse en esta dimensión. La finalización de la tarea individual y grupal, la implicación de todo el grupo es fundamental como evidencia puesto que este tipo de grupos suele caracterizarse por cierta tendencia a abandonar tareas largas o complejas, mucho más si son grupales.

\section{Dimensión mediacional}

- Recursos materiales: se utilizan materiales ricos en colores, formas tanto para la realización de cada gráfico como para la elaboración del mural final. La diversidad de materiales funciona a su vez como elemento motivador.

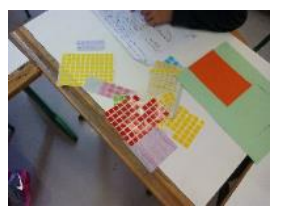

Figura 10. Folios de colores y pegatinas de colores

- Condiciones de aula y tiempo: el grupo es numeroso según sus características. Se utilizan sesiones lectivas de distintas asignaturas, disposiciones distintas de gestión de grupos (asamblea, pareja, secuencia) para aprovechamiento eficiente del tiempo.

\section{Dimensión interaccional}

- Interacción docente-discente: dicha interacción se centrado en la explicación adecuada del tema (presentación clara y bien organizada, énfasis en conceptos clave, etc.), la inclusión de todo el alumnado (resolviendo conflictos y realizando un reparto personalizado de las tareas para conseguir implicación) (Figura 5).

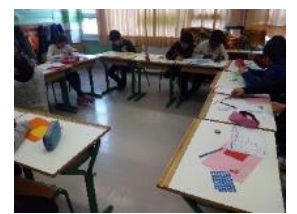

Figura 11. Reparto y realización de tareas individualizadas

- Interacción entre estudiantes: se actúa a nivel de favorecer el diálogo y comunicación entre estudiantes, de llegar a consensos, de inclusión en el grupo y de redefinición del uso de espacios (Figura 12).

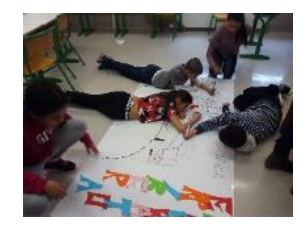

Figura 12. Redefinición del uso del aula para fomentar la colaboración 
- Autonomía: se hace seguimiento personalizado de cada estudiante, determinando su evolución mediante acciones relacionadas con la asunción de responsabilidad. Se hace hincapié cuando tienen que presentar los trabajos al grupo clase.

Este análisis pormenorizado de las actividades permite determinar para cada una de las dimensiones, qué componentes han sido desarrolladas (Tabla 6).

Tabla 6. Análisis de las actividades llevadas a cabo en el proyecto según las dimensiones de la ID y componente predominante en cada dimensión

\begin{tabular}{|c|c|c|c|c|c|c|}
\hline & Epistémica & Ecológica & Cognitiva & Afectiva & Interaccional & Mediacional \\
\hline A1 & & & & & $\begin{array}{l}\text { Interacción } \\
\text { docente- } \\
\text { discente (IDD) }\end{array}$ & \\
\hline A2 & & $\begin{array}{l}\text { Adaptación socio- } \\
\text { profesional y } \\
\text { cultural (ASP) }\end{array}$ & $\begin{array}{l}\text { Conocimientos } \\
\text { previos }(\mathrm{CP})\end{array}$ & $\begin{array}{l}\text { Intereses y } \\
\text { necesidades } \\
\text { (IN) }\end{array}$ & $\begin{array}{l}\text { Interacción } \\
\text { entre alumnos } \\
\text { (IA) }\end{array}$ & \\
\hline $\mathbf{A 3}$ & $\begin{array}{l}\text { Situación } \\
\text { problema }\end{array}$ & ASP & $\mathrm{CP}$ & IN & & \\
\hline A4 & & $\begin{array}{l}\text { Conexiones intra e } \\
\text { interdisciplinares } \\
\text { (CII) }\end{array}$ & & IN & IA & $\begin{array}{l}\text { Recursos } \\
\text { materiales } \\
(\mathrm{RM})\end{array}$ \\
\hline A5 & & CII & $\begin{array}{l}\text { Adaptaciones } \\
\text { curriculares a } \\
\text { diferencias } \\
\text { individuales } \\
\text { (ACU) }\end{array}$ & IN & $\begin{array}{l}\text { IA } \\
\text { IDD }\end{array}$ & \\
\hline A6 & & ASP & & $\begin{array}{l}\text { Actitudes } \\
\text { (AC) }\end{array}$ & IA & \\
\hline A7 & Lenguaje & & $\mathrm{CP}$ & $\mathrm{AC}$ & IA & $\mathrm{RM}$ \\
\hline A8 & $\begin{array}{l}\text { Reglas } \\
\text { (RE) }\end{array}$ & CII & $\mathrm{ACU}$ & $\mathrm{AC}$ & IA & \\
\hline A9 & $\mathrm{RE}$ & & $\mathrm{ACU}$ & IN & $\begin{array}{l}\text { IA } \\
\text { Autonomía }\end{array}$ & $\mathrm{RM}$ \\
\hline A10 & $\mathrm{RE}$ & & $\mathrm{ACU}$ & IN & $\begin{array}{l}\text { IDD } \\
\text { Evaluación } \\
\text { formativa }\end{array}$ & $\mathrm{RM}$ \\
\hline A11 & & & $\mathrm{ACU}$ & $\mathrm{AC}$ & IA & \\
\hline A12 & & CII & $\mathrm{ACU}$ & Emociones & IA & $\begin{array}{l}\text { RM/ } \\
\text { Condiciones } \\
\text { de aula }\end{array}$ \\
\hline
\end{tabular}

Para evaluar la idoneidad didáctica de acuerdo a las dimensiones propuestas en el EOS, se hace un estudio acerca de la aparición porcentual de las actividades por cada dimensión. Los resultados pueden leerse en la Tabla 7. Por otra parte, en los Anexos 1, 2, 34 y 5 se resumen aspectos de interpretación de datos para distintas dimensiones.

Tabla 7. Resumen de pesos relativos de las actividades según las dimensiones EOS

\begin{tabular}{cccc}
\hline Dimensión & AC/TOT & $\begin{array}{c}\% \text { aparición } \\
\text { actividad }\end{array}$ & $\begin{array}{c}\% \text { aparición } \\
\text { dimensión }\end{array}$ \\
\hline Epistémica & $5 / 12$ & $41.6 \%$ & $11.6 \%$ \\
Ecológica & $6 / 12$ & $50 \%$ & $14.2 \%$ \\
Cognitiva & $8 / 12$ & $66.6 \%$ & $18.6 \%$ \\
Afectiva & $10 / 12$ & $83.3 \%$ & $23.7 \%$ \\
Interaccional & $10 / 12$ & $83 \%$ & $21.3 \%$ \\
Mediacional & $5 / 12$ & $41.6 \%$ & $11.8 \%$ \\
\hline
\end{tabular}




\section{Conclusiones}

En este trabajo pretendíamos evaluar la idoneidad didáctica de un proyecto de estadística específicamente diseñado e implementado para $5^{\circ}$ de Educación Primaria de un centro con un alto porcentaje de familias en riesgo de exclusión social. Debido a su alta diversidad y, en muchos casos, dificultades con la lengua o el aprendizaje, el proyecto ha sufrido grandes adaptaciones curriculares, condición que influye en el desarrollo de los contenidos estadísticos exigidos en este curso, lo que sugiere una necesaria readaptación curricular de los contenidos en estos contextos de aprendizaje.

La falta de consecución en contenidos puramente estadísticos se ve compensada con la marcada intención interdisciplinar del proyecto, el cual ha introducido contenidos sobre demografía, uso del lenguaje en la elaboración de la encuesta y elementos propios de la competencia plástica en el informe-mural final. Esta interdisciplinaridad ha implicado un mayor grado de motivación en el alumnado (acorde con Anasagasti y Berciano, 2017; Berciano, Subinas y Anasagasti, 2020), dando lugar a una mayor participación y cambio actitudinal hacia la estadística (Siswono, Hartono y Kohar, 2018) y, así, se ha logrado la consecución de todas las tareas. La metodología propia del Aprendizaje Basado en Proyectos ha establecido en todo momento el vínculo entre el conocimiento de la estadística y su aplicabilidad con la realidad, fomentando el "sentido estadístico" en el alumnado (Batanero, Díaz, Contreras y Roa, 2013), puesto que los conceptos matemáticos implicados en el proceso han emergido a medida que el proyecto los ha requerido, de manera que su articulación con la realidad ha sido continua.

Los resultados desde el EOS corroboran estas ideas, en la medida en que todas las facetas que intervienen en un proceso de diseño e instrucción están representadas, aunque sus pesos relativos no son iguales. Como se recoge en investigaciones previas (Breda, Font y Pino-Fan, 2018), en contextos con alta diversidad de alumnado, se priorizan dimensiones como la afectiva en detrimento de la epistémica, a fin de fomentar la motivación e implicación de los estudiantes; esto lleva a un cierto desequilibrio entre el peso relativo de las dimensiones involucradas. En esta misma línea, investigaciones previas destacan la necesidad de conocer los indicadores asociados a la idoneidad afectiva en la enseñanza-aprendizaje de la matemática con el fin de evaluar la enseñanzaaprendizaje de la misma acorde a la EOS, y así poder mejorarla (por ejemplo, en el caso de la probabilidad, Beltrán-Pellicer y Godino, 2017).

En nuestro caso, el análisis de las dimensiones de la EOS permite concluir que la implementación de aula presenta una prevalencia de las dimensiones ecológica, cognitiva y afectiva en las primeras acciones relacionadas con la propuesta del proyecto. Este hecho viene marcado por el objetivo principal de fomentar la implicación del alumnado; planteando una situación problema, en la que se percibiera la utilidad de la estadística para la descripción del barrio, potenciando así la conexión del proyecto con su entorno, con otras asignaturas y con sus conocimientos previos. Una vez establecida esa primera aproximación, las dimensiones interaccional y mediacional cobran protagonismo, ya que se establecen dinámicas de aula que promueven el intercambio de conocimiento entre estudiantes, para la interpretación y construcción de gráficos, situaciones necesarias para que aparezcan conflictos semióticos asociados a la naturaleza de los gráficos y su aplicabilidad a la vida real, con el fin de generar una cultura estadística en los niños y niñas (Arteaga, Batanero y Contreras, 2011).

A pesar de este desequilibrio en el logro de una idoneidad alta en las distintas dimensiones del EOS, este proyecto ha promovido un aprendizaje significativo, no sólo en el área de ciencias sociales, sino en las referentes a contenidos estadísticos básicos. 
El éxito del proyecto en su globalidad se refiere a la idoneidad ecológica, afectiva, interaccional y mediacional, ya que se ha promovido la motivación en el alumnado y la creación de "sentido estadístico" en torno a la tarea asociada.

\section{Agradecimientos}

Ayudas a la investigación de la Universidad del País Vasco UPV/EHU: Mod. II Grupos (códigos GIU19/008 y PPGA20/14).

\section{Referencias}

Anasagasti, J. y Berciano, A. (2017). Estadística y ABP. Una experiencia con futuro profesorado de primaria. UNO, 78, 18-23.

Arteaga, P., Batanero, C. y Gea, M. M. (2017). La componente mediacional del conocimiento didáctico-matemático de futuros profesores sobre estadística: Un estudio de evaluación exploratorio. Educação Matemática Debate, 1(1), 54-75.

Arteaga, P., Batanero, C. y Contreras, J. M. (2011). Gráficos estadísticos en la educación Primaria y en la Formación de Profesores. Boletín de Estudios en Investigación, 12, 123-135.

Batanero, C. y Díaz, C. (2004). El papel de los proyectos en la enseñanza y aprendizaje de la estadística. En J. P. Royo (Ed.), Aspectos didácticos de las matemáticas (pp. 125-164). Zaragoza: ICE.

Batanero, C. y Díaz, C. (Eds.) (2011). Estadística con proyectos. Granada: Universidad de Granada.

Batanero, C., Díaz, C., Contreras, J. M. y Roa, R. (2013). El sentido estadístico y su desarrollo. Números, 83, 7-18.

Beltrán-Pellicer, P. y Godino, J. D. (2017). Aplicación de indicadores de idoneidad afectiva en un proceso de enseñanza de probabilidad en educación secundaria. Perspectiva Educacional, 56(2), 92-116.

Berciano, A., Subinas, A. y Anasagasti J. (2020). Estadística basada en proyectos en contextos de riesgo de exclusión social: Cómo inferir esta práctica a la formación inicial del profesorado. En M. M. Gea., R. Álvarez-Arroyo y J. A. Garzón (Eds.), Seminario Hispano Brasileño de Educación Estadística (pp. 25-28). Granada: Grupo PAI FQM-126.

Breda, A., Font, V. y Pino-Fan, L. R. (2018). Criterios valorativos y normativos en didáctica de las matemáticas: El caso del constructo idoneidad didáctica. Bolema, 32(60), 255-278.

Breda, A., Pino-Fan, L. y Font, V. (2017). Meta didactic-mathematical knowledge of teachers: Criteria for the reflection and assessment on teaching practice. Eurasia Journal of Mathematics, Science \& Technology Education, 13(6), 1893-1918.

Decreto 236/2015, de 22 de diciembre, por el que se establece el currículo de la Educación Básica y se implanta en la Comunidad Autónoma del País Vasco. Boletín Oficial del País Vasco. Vitoria, 15 de enero de 2016. Recuperado 15/05/2019 de https://www.euskadi.eus/decretos-curriculares-de-la-capv/web01-a3hbhezi/es

Font, V., Planas, N. y Godino, J. D. (2010). Modelo para el análisis didáctico en educación matemática. Infancia y Aprendizaje, 33(2), 89-105. 
Friel, S. N., Curcio, F. R. y Bright, G. W. (2001). Making sense of graphs: Critical factors influencing comprehension and instructional implications. Journal for Research in mathematics Education, 32(2), 124-158.

García-Valcárcel, A. y Basilotta, V. (2017). Aprendizaje Basado en Proyectos (ABP): evaluación desde la perspectiva de alumnos de Educación Primaria. Revista de Investigación Educativa, 35(1), 113- 131.

Godino, J. D. (2013). Indicadores de la idoneidad didáctica de procesos de enseñanza y aprendizaje de las matemáticas. Cuadernos de Investigación y Formación en Educación Matemática, 11, 111-132.

Godino, J. D. Batanero, C. y Font, V. (2007). The ontosemiotic approach to research in mathematics education. ZDM, 39(1-2), 127-135.

Godino, J. D., Contreras, A. y Font, V. (2006). Análisis de procesos de instrucción basado en el enfoque ontológicosemiótico de la cognición matemática. Recherches en Didactiques des Mathematiques, 26(1), 39-88.

Godino, J. D., Wilhelmi, M. R. y Bencomo, D. (2005). Suitability criteria of a mathematical instruction process. A teaching experience of the function notion. Mediterranean Journal for Research in Mathematics Education, 4(2), 1-26.

Gómez, D. y Martínez, J. M. (1997). La evaluación en Ciencias Sociales: la Historia Económica. Boletín del Instituto de Estudios Almerienses, 15,133-146.

Instituto Nacional de Evaluación Educativa [INEE] (2015). TIMSS 2015. Estudio internacional de tendencias en Ciencias y Matemáticas. IEA. Informe español: Resultados y contexto. Madrid: Ministerio de Educación, Cultura y Deporte.

Irakas Sistema Ebaluatu eta Ikertzeko Erakundea [ISEI-IVEI] (2014). Nivel de competencias curriculares en $4^{\circ}$ curso de educación primaria: Matemática. Bilbao: ISEI-IVEI.

Koparan, T. y Güven, B. (2014). The effect of Project based learning on the Statistical Literacy levels of student 8th grade. European Journal of Educational Research, 3(3), 145-157.

Mergendoller, J. R., Maxwell, N. L. y Bellisimo, Y. (2006). The effectiveness of problem-based instruction: A comparative study of instructional methods and student characteristics. Interdisciplinary Journal of Problem-based Learning, 1(2), 49-69.

Molina, M., Castro, E., Molina, J. L. y Castro, E. (2011). Un acercamiento a la investigación de diseño a través de los experimentos de enseñanza. Enseñanza de las Ciencias, 29(1), 75-88.

Nogueira, I. C., Fernández, T. y Rodríguez, D. (2015). Análise Ontossemiótica de processos de instruçao matemática - um exemplo no Ensino Básico. Saber \& Educar, 20,174-187.

OECD (2019). PISA 2018 Results (Volume II): Where all students can succeed. París: OECD.

Planas, N. (2010). Las teorías socioculturales en la investigación en educación matemática: Reflexiones y datos bibliométricos. En M. M. Moreno, A. Estrada, J. Carrillo y T. A. Sierra, (Eds.), Investigación en Educación Matemática XIV (pp. 163195). Lleida: SEIEM 
Pozuelos, J. y Rodríguez, F. (2008). Trabajando por proyectos en el aula. Aportaciones de una investigación colaborativa. Investigación en la Escuela, 66, 5-27.

Ramos, A. B., Fagúndez, Z, T. y Castells, M. (2009). Los criterios de idoneidad y propuestas de cambios institucionales en el ámbito universitario. Investigación y Postgrado, 24(3), 115-139.

Siswono, T. Y. E., Hartono, S. y Kohar, A.W. (2018). Effectiveness of project based learning in statistics for lower secondary schools. Eurasian Journal of Educational Research, 75, 197-210.

Subinas, A. y Berciano, A. (2019). La motivación en el aula de matemáticas: ejemplo de yincana en $5^{\circ}$ de Educación Primaria. Números, 101, 45-58.

Tarmizi, R. A. y Bayat, S. (2010). Effects of problem-based learning approach in learning of statistics among university students. Procedia. Social and Behavioral Sciences, 8, 384-392.

Thomas, J. (2000). A review of research on project-based learning. San Rafael, CA: Autodesk Foundation.

Wild, C. y Pfannkuch, M. (1999). Statistical thinking in empirical enquiry. International Statistical Review, 67(3), 223-265.

\section{Referencias de los autores}

Ainhoa Berciano, Universidad del País Vasco/Euskal Herriko Unibertsitatea. ainhoa.berciano@ehu.eus

Ainhoa Subinas, Universidad del País Vasco/Euskal Herriko Unibertsitatea. ainhoa.subinas@ehu.eus

Jon Anasagasti, Universidad del País Vasco/Euskal Herriko Unibertsitatea. jon.anasagasti@ehu.eus 


\title{
Analysis of the didactic suitability of a statistical project designed and implemented in a context at risk of social exclusion
}

\author{
Ainhoa Berciano, Universidad del País Vasco/Euskal Herriko Unibertsitatea \\ Ainhoa Subinas, Universidad del País Vasco/Euskal Herriko Unibertsitatea \\ Jon Anasagasti, Universidad del País Vasco/ Euskal Herriko Unibertsitatea
}

\begin{abstract}
Statistical education must respond to the challenge of how to foster critical thinking in students. Such a challenge becomes singularized in contexts at risk of social exclusion, and hence studies that evaluate the didactic suitability of different types of education are key. Our interest is focused on analyzing a statistical project specifically designed for a socioeconomically complex school context of primary education. After the project implementation in a 5th grade classroom, we analyzed: 1) the work around curricular contents and 2) its didactic suitability according to theoretically-established dimensions. Overall, our project relates academic work with life situations such as visiting the market in the neighborhood, researching population measures and drawing on statistic tools to describe them accurately. The analytical methods were mixed and inspired by the Ontosemiotic Approach in mathematics education research. We classified the project tasks from a qualitative point of view, and we carried out a quantitative study to determine the relative weight of the dimensions of didactic suitability according to our theory of reference. We are able to conclude that the project satisfied the required conditions to motivate students and to improve statistical knowledge. We must, nonetheless, note some imbalance in the achievement level of the didactic suitability dimensions. From the analyses, the least represented dimensions appear to be the epistemic and the mediational, while those most representative are the ecological, the cognitive, the affective and the interactional. We conclude thus about the need to address a certain balance amongst the least and the most represented dimensions. Since the main goal of the project was to involve students in the tasks, and relate the contents of social sciences and mathematics to real situations, this could explain the weak epistemic achievement around statistical contents in favor of global processes of comprehension of contents as applied and connected to other content subjects and daily life situations.
\end{abstract}


Anexo 1. DIMENSIÓN EPISTÉMICA

Tabla 8. Análisis de las actividades llevadas a cabo en el proyecto y componente, evidencias y justificación de ausencias

\begin{tabular}{|c|c|c|c|}
\hline COMPONENTES & ACCIONES & DESCRIPCIÓN & EVIDENCIA \\
\hline Situación problema & A3 & $\begin{array}{l}\text { Se problematiza la unidad didáctica sobre la población, la dificultad de describir sus } \\
\text { características y la necesidad de un método de investigación para obtener datos. }\end{array}$ & \\
\hline Lenguaje & A7 & $\begin{array}{l}\text { Las gráficas expuestas para practicar la interpretación de gráficas son muy sencillas, } \\
\text { las preguntas se secuencian según los niveles de comprensión de gráficos y se incide } \\
\text { menos en los niveles críticos o superiores. }\end{array}$ & Aillili. \\
\hline Reglas & $\mathrm{A} 8, \mathrm{~A} 9, \mathrm{~A} 10$ & $\begin{array}{l}\text { Se proponen pautas muy adaptadas: utilización de pegatinas cuadradas para la } \\
\text { representación de las frecuencias absolutas, de modo que se provee de una } \\
\text { representación proporcional directa. }\end{array}$ & \\
\hline Argumentos & & $\begin{array}{l}\text { No se priorizan situaciones de generar o negociar definiciones, proposiciones o } \\
\text { procedimientos; hay un énfasis en la comprensión más cualitativa y global. }\end{array}$ & $\begin{array}{l}\text { No se definen ni enseñan conceptos teóricos estadísticos durante } \\
\text { la unidad didáctica a fin de no abstraer demasiado. }\end{array}$ \\
\hline Relaciones & & Se trata de evitar definiciones, y proposiciones que puedan resultar demasiado & $\begin{array}{l}\text { La ausencia de contenido puramente matemático se explica desde } \\
\text { la prioridad de tratar el tema estadístico desde una perspectiva } \\
\text { intuitiva, de ahí la ausencia de definiciones y proposiciones. }\end{array}$ \\
\hline
\end{tabular}

Anexo 2. DIMENSIÓN ECOLÓGICA

Tabla 9. Análisis de las actividades llevadas a cabo en el proyecto y componente, evidencias y justificación de ausencias

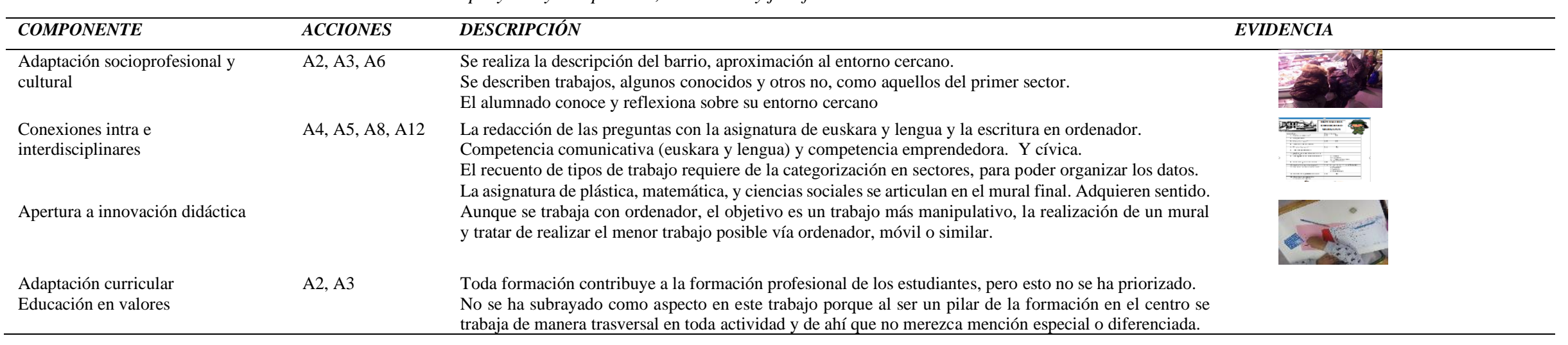

Anexo 3. DIMENSIÓN COGNITIVA 
Tabla 10. Análisis de las actividades llevadas a cabo en el proyecto y componente, evidencias y justificación de ausencias

\begin{tabular}{lll}
\hline COMPONENTE & ACCIONES & DESCRIPCIÓN \\
\hline Conocimientos previos & A2,A3,A7 & $\begin{array}{l}\text { Descripción de ideas estereotipos. Se articulan mediante contenidos de ciencias sociales e ideas previas. } \\
\text { Las gráficas están adaptadas para poder describirlas de manera intuitiva }\end{array}$ \\
$\begin{array}{l}\text { Adaptaciones curriculares a } \\
\text { diferencias individuales }\end{array}$ & A5, A8, A9, & $\begin{array}{l}\text { Existe un alumno con más facilidad que se ocupa de la explicación del histograma. } \\
\text { Participación en el mural de acuerdo a las capacidades e intereses del alumnado }\end{array}$ \\
Aprendizaje & & \\
\hline
\end{tabular}

\section{Anexo 4. DIMENSIÓN AFECTIVA}

Tabla 11. Análisis de las actividades llevadas a cabo en el proyecto y componente, evidencias y justificación de ausencias

\begin{tabular}{lll}
\hline COMPONENTE & ACCIONES & DESCRIPCIÓN \\
\hline Intereses y necesidades & A2, A3, A4, A5, & $\begin{array}{l}\text { La presentación de la situación problema y su relación con la estadística } \\
\text { La sesión de preguntas es abierta. Cada alumno/a pregunta en función de sus intereses. } \\
\text { Se reconduce la sesión anterior y se consensuan preguntas atendiendo a las necesidades de tipos de variables } \\
\text { La distribución de tareas permite diferentes niveles de implicación, pero a su vez sean todas imprescindibles } \\
\text { en el desempeño de la tarea global. } \\
\text { La presentación de la situación problema y su relación con la estadística } \\
\text { Ejercicios de interpretación de graficas adaptados a los conocimientos previos que permiten la participación } \\
\text { y acercamiento a los conceptos por parte de todo el alumnado La distribución de tareas permite diferentes } \\
\text { niveles de implicación, pero a su vez sean todas imprescindibles en el desempeño de la tarea global. }\end{array}$ \\
Actitudes & A6, A7, A8, A12 & \\
Emociones & A12
\end{tabular}

Anexo 5. DIMENSIÓN MEDIACIONAL

Tabla 12. Análisis de las actividades llevadas a cabo en el proyecto y componente, evidencias y justificación de ausencias

\begin{tabular}{ll} 
COMPONENTE & ACCIONES \\
\hline Recursos materiales & $\begin{array}{l}\text { A4, A7, A9, } \\
\text { A10, A11 }\end{array}$
\end{tabular}
A4, A7, A9,
A10, A11

Condiciones de aula

Tiempo

\section{DESCRIPCIÓN}

Se utiliza el ordenador para el diseño de las encuestas Se utiliza la pantalla digital para la presentación de los ejercicios de interpretación de gráficas.

Se utilizan materiales atractivos de papelería: hojas de colores, pegatinas de colores, etc.

No se contemplan otros indicadores por no tener capacidad para modificarlos. Tanto número y distribución de alumnos como horario no son variables a gestionar porque no se dispone de potestad para ello.

Se alternan configuraciones para distribuir el alumnado de modos diferentes por atividad y optimizar rendimiento.

Los tiempos también se gestionan en función de las necesidades, pero la limitación tiene más que ver con capacidad de

Los tiempos también se gestionan en función de las necesidades, pe
atención a una tarea que a la complicación del tema a desarrollar.

Se utilizan sesiones del área de plástica para la construcción del mural.

\section{EVIDENCIA}

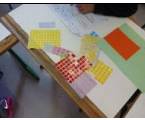

